\title{
Ad occhi aperti verso il futuro. Il ruolo del digital storytelling nella progettazione partecipata di percorsi di orientamento
}

\author{
di Antonio Borgogni ${ }^{\circ}$ Marco Lazzari ${ }^{\wedge}$ Alice Ponzoni ${ }^{\wedge}$ Stefano Tomelleri* ${ }^{\wedge}$
}

\section{Riassunto}

Il progetto qui descritto è stato pensato per supportare, attraverso azioni di tutoraggio tra pari basate sul digital storytelling, studenti provenienti in particolare da istituti superiori professionali nell'orientamento verso i Corsi di studio in Scienze dell'educazione.

Il progetto si è basato su due principali costrutti teorici. L'approccio fenomenologico, riflettendo sulla progettazione esistenziale autentica e inautentica e discutendo sulle parole scelta e differenza. Il paradigma della complessità, ragionando in opposizione al sovrano sotterraneo costituito dal paradigma della semplificazione.

Oltre a ciò, l'orientamento universitario viene presentato anche sul piano della rilevanza politica alla luce del basso indice di mobilità sociale del nostro Paese che colpisce anche coloro, qui nominati metafragili - in qualità di diplomati desiderosi di ottenere una laurea, che difficilmente potranno raggiungere livelli di antifragilità.

Le azioni messe in atto (tutoraggio, orientamento tramite le tecnologie, autovalutazione delle capacità di svolgere le professioni in uscita, formazione per gli studenti e i tutor universitari) e i significati dell'approccio narrativo basato sul digital storytelling sono stati rivisitati concentrandosi sulle relazioni tra aspettative e contesto organizzativo alla luce dell'irriducibilità semiotica di cui la narrazione è portatrice.

\footnotetext{
○Università degli Studi di Bergamo. Corresponding author: antonio.borgogni@unibg.it. ${ }^{\wedge}$ Università degli Studi di Bergamo.

* Gli autori, citati in ordine alfabetico, hanno congiuntamente curato e revisionato il testo. In particolare: Antonio Borgogni ha curato i paragrafi sulla progettazione esistenziale e sulla trasformazione organizzativa; Marco Lazzari ha concettualizzato e supervisionato la stesura, curato il paragrafo sulla descrizione del percorso progettuale e le conclusioni; Alice Ponzoni ha curato il paragrafo sulla descrizione del percorso progettuale; Stefano Tomelleri ha curato l'introduzione e il paragrafo sulla metodologia.
}

Educational Reflective Practices (ISSNe 2279-9605), 1/2021

Doi: 10.3280/erpoa1-2021oa11520 
Nelle conclusioni, il percorso progettuale è stato ricontestualizzato nell'attuale emergenza pandemica focalizzandosi sulle opportunità di apprendimento trasformativo dell'organizzazione universitaria.

Parole chiave: orientamento universitario; digital storytelling; narrazione; progettazione esistenziale; complessità; apprendimento trasformativo.

\section{Abstract. Open-eyed towards the future. The role of digital storytelling in participatory planning of guidance pathways}

The project described in this contribution has been planned to support, through a peer tutoring based on digital storytelling, students coming from vocational upper secondary schools to find their pathways in the Educational Sciences bachelor's degree course.

The project has been grounded on two main conceptual frames. The phenomenological approach, reflecting on authentic and unauthentic existential planning and discussing the words choice and difference. The paradigm of complexity, debating against the underground ruler represented by the paradigm of simplification.

Therefore, the university guidance is presented in its political relevance in the light of the low grade of social mobility in Italy, which is affecting those, here named meta-frails - as school-leaver aiming at university bachelor's degree, who unlikely could achieve antifragility.

The actions (tutoring, guidance through ICTs, self-assessment of the attitudes towards the placement, training for the students and for the university's tutors) and the meanings of the narrative approach through digital storytelling have been reviewed focusing on the link between organizational context and expectations in the light of the semiotic irreducibility of the narration.

In conclusion, the entire pathway of the project has been recontextualized in the actual pandemic emergency focusing on the opportunities for a transformative organizational learning of the university.

Keywords: university guidance; digital storytelling; narrative; existential planning; complexity; transformative learning

First submission: 15/02/2021, accepted: 28/02/2021

Available online: $27 / 04 / 2021$

\section{Introduzione}

Il progetto Ad occhi aperti verso il futuro, realizzato nell' ambito del corso 
di studi in Scienze dell'educazione dell'Università degli studi di Bergamo, fa parte del progetto Percorsi di orientamento e tutorato per promuovere il successo universitario e professionale (SUPER), finanziato dal MIUR per l'AA 2018/19'.

Al momento dello svolgimento del progetto, il Corso di studi si articolava in cinque curricula: educatore nei servizi sociali e di comunità, nei servizi per la prima infanzia, nei servizi per gli anziani e nei servizi per il lavoro, nei servizi per le attività motorie e sportive. Il corso di laurea prevede, per tutti gli indirizzi curriculari, la realizzazione obbligatoria di un tirocinio per sperimentare in modo riflessivo, e con la partecipazione attiva degli stakeholder, il lavoro che si svolge nelle diverse organizzazioni.

Il progetto di orientamento e tutorato, coerentemente con l'architettura del corso di studi, ha previsto che le attività di progettazione, ricerca e monitoraggio fossero realizzate in stretta collaborazione con i docenti delle scuole superiori e gli enti territoriali. Nel corso dell'intero progetto sono stati realizzativi workshop periodici per la definizione del disegno del percorso di orientamento e tutoraggio e del suo monitoraggio in itinere.

Il percorso si è focalizzato in particolare sulle studentesse degli istituti professionali e dei licei delle scienze umane e sociali poiché gli iscritti al corso di studi, come si evince dalla Tabella 1, sono prevalentemente donne $(89 \%)$ provenienti dagli istituti professionali $(39,6 \%)$ e da licei delle scienze umane $(28 \%)$.

Tab. 1 - Immatricolati 2018-2019 per tipo di diploma (Fonte Esse 3 Unibg)

\begin{tabular}{llll}
\hline Tipo di diploma & F & M & Tot \\
\hline Diploma estero & 0 & 0 & 0 \\
Liceo classico & $0,9 \%$ & $0,4 \%$ & $1,3 \%$ \\
Liceo scientifico & $4,5 \%$ & $1,3 \%$ & $5,8 \%$ \\
Liceo linguistico & $2,4 \%$ & $0,1 \%$ & $2,6 \%$ \\
Liceo delle scienze umane & $28 \%$ & $2,1 \%$ & $30 \%$ \\
Istituto tecnico & $9,0 \%$ & $3,0 \%$ & $12 \%$ \\
Istituto professionale & $39,6 \%$ & $3,4 \%$ & $43 \%$ \\
Altro & $4,2 \%$ & $0,5 \%$ & $4,7 \%$ \\
Non specificato & $0,4 \%$ & $0,3 \%$ & $0,6 \%$ \\
Totale & $89 \%$ & $11 \%$ & $100 \%$ \\
\hline
\end{tabular}

Sebbene negli ultimi anni la percentuale di studenti regolarmente iscritti (considerando tali gli iscritti entro la durata normale del corso di laurea, con almeno 40 CFU acquisiti nell'A.A.) sia costante e intorno al 60\%, si è ipotizzato che in particolare le studentesse degli istituti professionali, per le specificità degli istituti di provenienza, fossero più orientate alla scelta

\footnotetext{
${ }^{1}$ Il progetto ha avuto come capofila l'Università degli studi di Siena, Dipartimento di Scienze della Formazione, Scienze Umane e della Comunicazione Interculturale.
} 
professionale che al proseguimento degli studi universitari e richiedessero perciò un percorso di orientamento in entrata e di tutoraggio in itinere. Inoltre, pur sapendo che il dato relativo alla percentuale dei laureati occupati a un anno dalla laurea è dell' $85,2 \%$ (iC06TER, dato relativo ai laureati del 2017, fonte AVA-SUA 2018-2019) e che tale percentuale è superiore alle percentuali rilevate a livello di area geografica di appartenenza e a livello nazionale, sulla base dell'esperienza didattica maturata negli anni si è ipotizzato che le studentesse degli istituti professionali, comparativamente alle studentesse provenienti dai licei, fossero culturalmente e socialmente più fragili ("metafragili" come argomenteremo in seguito), richiedendo perciò un appropriato percorso di riflessività e consapevolezza della scelta universitaria in relazione alle opportunità lavorative e agli sviluppi di carriera.

\section{L'orientamento come progettazione esistenziale: il ruolo dell'edu- cazione}

Tra le categorie pedagogiche in grado di accompagnare questo contributo, facciamo qui riferimento alle riflessioni relative alla progettazione esistenziale come approfondite in particolare da Giovanni Maria Bertin (Bertin \& Contini, 1983). Gli autori individuano gli antecedenti storico-culturali della progettazione esistenziale a partire dall'esistenzialismo che la intende, in senso lato, come l'anticipazione di possibilità (Bertin \& Contini, 1983) e che qui sintetizziamo nella riflessione sartriana sulla scelta: «nel riconoscimento che ho libertà di scegliere, ma non libertà di non scegliere perché non scegliere "vuol dire scegliere di non scegliere" (Sartre, 1942 in Bertin \& Contini, 1983, p. 90). E se la scelta è immanente alla vita, allora «la progettazione $[\ldots]$ come l'orientamento [...] del soggetto [...] rivolto ad elaborare, vagliare ed unificare aspirazioni, criteri di valore ed obiettivi $[\ldots]$ non in funzione dell'attuale (dell'esistente) ma in funzione del possibile (dell'esistenziale)» (Bertin \& Contini, 1983, pp. 90-91).

Gli orizzonti di senso aperti da una visione siffatta dell'orientamento pur non inteso da parte di Bertin e Contini (1983) nel senso di compito accademico ma dell'educazione lato sensu - sono, a nostro parere, assai vasti, attualissimi e propri di quella capacità della filosofia, non sempre presente né praticabile, di declinarsi nelle fenomenologie quotidiane.

La scelta o piuttosto il sistema di scelte secondo cui essa si articola nell'arco della vita, è, pertanto, elemento decisivo della progettazione che dovrà confrontarsi con altre scelte possibili. 
Compito dell'azione educativa, ancora secondo Bertin e Contini (1983), è di aiutare il soggetto a discriminare tra progettazione autentica e inautentica alla luce della categoria della differenza.

La progettazione sarà autentica, rigorosa e ricca, se basata sul confronto approfondito con le altre scelte possibili, sulla «riflessione critica su esperienze e prospettive esistenziali» (Bertin \& Contini, 1983, p. 91); sarà inautentica quando quel confronto sarà povero e superficiale.

Ma anche la progettazione autentica può essere sconvolta da «eventi imprevisti o dalla caduta di valori esistenziali» (p. 91) e qui il ruolo educativo si esplica nell'aiuto ad evitare di precipitare nella ricerca dell'alibi o, al contrario, nella colpevolizzazione.

Ci spingeremmo oggi a parlare di una sorta di impalcatura, di scaffolding esistenziale, di struttura, leggera, strutturata e al contempo strutturante, che temporaneamente spinge con gentilezza al superamento dell'impasse.

La progettazione inautentica è, sovente, improntata all'acriticità, al velleitarismo, alla incoerenza, alla unilateralità ed è qui, interpreteremmo, ancor più chiara la necessità, e la difficoltà, dell'educazione, a rintracciare queste debolezze o false forze per riportale nel tracciato della progettazione esistenziale.

Ma anche la progettazione autentica sarà possibile quando avrà come obiettivo principale il momento della differenza: «dell'attitudine ad effettuare, in ogni aspetto del quotidiano, il superamento dei condizionamenti psicologici, sociopolitici e culturali da cui egli è pressato nella sua realtà storica» (p. 92). La differenza è un criterio della progettazione esistenziale e oppone «sul piano del possibile, la complessità dei valori alla semplificazione, alla riduzione, all'elementarizzazione [...] è caratterizzata dal suo opporre l'altezza e la profondità a ciò che è basso e superficiale» (p.94).

E l'attualità e urgenza delle parole di Bertin e Contini (1983), la loro pregnanza educativa, sociale e politica, risaltano alla luce di almeno due riflessioni: sul linguaggio e sulla complessità.

La riflessione sul linguaggio fa riferimento a quella di Liliana Segre, proposta in varie occasioni e qui tratta da una trasmissione radiofonica:

Quali parole lei vorrebbe vedere espulse dal linguaggio (chiede il conduttore Davide Assael)? Dire che cosa togliere [è assai difficile] vorrei dire piuttosto che cosa mettere in quel vocabolario così scarso in cui gli stereotipi di ciò che si sente per la strada, di ciò che si sente in metropolitana, della povertà del linguaggio, della schematizzazione modaiola, di alcuni modi di dire che condizionano profondamente la società $[\ldots]$ parole (da togliere) sarebbero certamente antisemitismo, razzismo, $[\ldots]$ odio, torturare, umiliare. E quelle che vogliamo mettere? Mettere nel vocabolario? Quali sono? Scelta, differenza, amore, sono poche quelle da mettere, sono 
pochissime, sono una specie di abc della civiltà $[\ldots]$ non sono più usate, non sono di moda» (Radio 3, Uomini e profeti, 31 gennaio 2021).

Sentiamo qui l'urgenza, nel tentativo di attualizzare il pensiero di Bertin e proprio in vista della seconda riflessione, di rimarcare come, a livello educativo, sia sempre più necessario intervenire su coloro che non vengono intercettati dall'istituzione e coloro che lo sono debolmente che, pur presenti, sono in una zona grigia che è in pericolo rispetto all'inautenticità e semplificazione, esposta a quelle "parole da togliere" e a cui manca la pratica delle "parole da mettere". Parliamo di ragazze e ragazzi per varie ragioni in una situazione di disagio, sociale o culturale, non così profonda, che se la "sono cavata" nella scuola ma che, affacciandosi al mondo universitario, riportando a livello accademico la metafora di Canevaro (1976), si "perdono nel bosco" soprattutto nei primi tempi, tra gli sterpi e i rovi che, a volte, ne costituiscono il limitare.

Proprio questo è il senso, in fondo, del progetto di orientamento che stiamo descrivendo, condiviso con le altre università nella ricchezza delle declinazioni locali.

La seconda riflessione rimanda alla complessità, tema istitutivo del Dipartimento cui appartengono gli autori del presente contributo. La complessità dei sistemi e dei fattori che gravano sui percorsi degli studenti, su quella che diventa, in particolare nei momenti di passaggio, una sorta di ricercaazione esistenziale (Barbiér, 1983, 1996), e sulle scelte e conseguenti differenze, ci aiuta ad attualizzare le riflessioni sopra esposte. Una progettazione autentica, come ci ricorda Bertin, comporta la possibilità di una visione ampia evitando, per quanto possibile, i rischi del sovrano sotterraneo ovvero, secondo Ceruti (2020) il "paradigma della semplificazione", il «paradigma che ha preteso di risolvere e dissolvere la complessità con la semplificazione per mezzo della linearità causa-effetto, della scomposizione in unità elementari della realtà, dell'isolamento dell'oggetto dal suo contesto» (Ceruti \& Bellusci, 2020, p.64). Ma in un'ottica di educazione che deve fare i conti con la globalizzazione (Bocchi \& Ceruti, 2004), l'educazione deve imperniarsi sulla capacità di connettere conoscenze, di «distinguere e articolare logiche differenti $[\ldots]$ concependo i confini tra le discipline $[\ldots]$ come aree di interazione, spazi intermedi dove nascono i problemi più interessanti, gli approcci più originali» (Ceruti \& Bellusci, 2020, p. 144). La scuola, che deve insegnare a concepire «l'unità nella diversità umana e la diversità nell'unità umana [...] è un luogo di cura dell'anima, di socialità, di esercizio all'attenzione, di sviluppo vocazionale, [...] di viatico alla vita personale e civile» (p. 145). 


\section{L'orientamento come fattore politico: la trasformazione organiz- zativa}

In un Paese che presenta profonde difficoltà sul piano della mobilità sociale, come evidenzia Openpolis (2020) anche elaborando recenti dati del World Economic Forum e dell'OCSE, l'orientamento assume, ancor più che altrove, una chiara connotazione politica: scavalca il concetto di bussola pur di grande rilevanza - facendo assumere all'orientamento un ruolo prefigurativo - narrando il territorio ancor più che indicando direzioni o dispiegando una mappa - rispetto agli scenari di vita. Le disuguaglianze, come ben evidenzia anche Save the children (2020), hanno un'origine profondamente radicata nell'infanzia e nella condizione socioeconomica della famiglia.

Nel bosco accademico in cui si addentrano le ragazze e i ragazzi appena maggiorenni si perdono i metafragili, coloro che sono comunque riusciti ad arrivare al mare ma che non sanno nuotare, o non possono dotarsi di strumenti per navigarlo; non diventeranno, o difficilmente diventeranno, antifragili (Taleb, 2013). Non saranno in grado di trarre vantaggio dagli accadimenti casuali, di riflettere sui propri elementi di forza, di trasformare lo stress e le deviazioni di percorso in risorsa.

E qui si pone il tema di quanto l'università si ponga il problema e di come intenda risolverlo. Il presente progetto POT è profondamente radicato nelle teorie sulla trasformazione (Mezirow, 2016) e si esplicita come la trasposizione di logiche e metodi trasformativi a livello organizzativo (Fabbri \& Romano, 2017).

Il progetto bergamasco, di seguito descritto sul piano metodologico, ha individuato alcuni criteri, in particolare l'educazione tra pari, e strumenti, il digital storytelling, ma ha anche fatto riflettere, grazie alle presentazioni, ai contatti, agli incontri della commissione orientamento del Dipartimento, rispetto ad un bisogno che riguarda anche gli altri Corsi di Studio. L'edizione del POT 2019/20 (DM 435 del 6 agosto 2020) si concentra, infatti, proprio sul sistema di tutoraggio tra pari rivolto in particolare alle matricole. Nel caso del Dipartimento degli autori del presente contributo, il nuovo progetto non solo si basa sull'esperienza precedente ma vede anche momenti di formazione comune tra studenti coinvolti nel POT 2018/19 e i tutor di quello attuale.

\section{La metodologia: l'analisi delle narrazioni e il digital storytelling nella progettazione partecipata}

L'accompagnamento riflessivo delle studentesse e degli studenti 
all'interno del progetto bergamasco si è basato sull'analisi delle narrazioni che si sono dispiegate nei passaggi, nelle iniziazioni, nelle difficoltà e nelle risorse incontrate dagli studenti nella navigazione all'interno della vita accademica a Bergamo.

In una prima fase il percorso di orientamento ha previsto la costruzione condivisa, in un workshop dedicato cui hanno partecipato enti territoriali, docenti delle scuole superiori e docenti universitari, di un repertorio di temi chiave (aspettative professionali, metodo di studio, ambiente universitario, autonomia e autorganizzazione nello stile di vita universitario) da sottoporre alle studentesse nel corso di quattro focus group. I quattro focus group erano composti da due gruppi misti da 15 e da 11 studenti $^{2}$.

L'analisi dei focus group ha evidenziato una profonda differenza tra le narrazioni delle studentesse provenienti dal Liceo, e quelle delle studentesse provenienti dagli Istituti Professionali. Le narrazioni dei ragazzi e delle ragazze del Liceo hanno visto come protagonista il desiderio, declinato principalmente come desiderio di scoperta/curiosità verso l'ambiente universitario e le nuove discipline che l'università propone. Nei loro racconti è emersa la speranza e il desiderio di mettersi alla prova, sperimentando quell'autonomia che l'università richiede, anche attraverso l'autogestione e l'organizzazione degli esami e del carico di studio. Al contrario le studentesse provenienti dall'Istituto professionale hanno adottato prevalentemente un registro narrativo caratterizzato da un sentimento di inadeguatezza e dalla paura verso il mondo universitario definito come sconosciuto.

In seguito, il percorso ha previsto le seguenti attività:

1) Percorsi di didattica tutoriale. In questa fase gli studenti della scuola secondaria sono stati coinvolti da parte dei tutor studenti universitari nella costruzione degli strumenti di ricerca, attraverso una serie di incontri in cui era prevista la produzione di tutorial (utilizzati poi negli incontri previsti con le classi della Scuola di appartenenza) e di alcuni storytelling. In questa fase sono stati coinvolti anche i partner territoriali che hanno dato il loro contributo nel far conoscere al meglio gli ambiti lavorativi relativi ai profili professionali.

2) Percorsi di orientamento attraverso le nuove tecnologie: il progetto si è basato sulla produzione di materiali filmati. Per questo i tutor hanno avuto il compito di comprendere le competenze digitali degli studenti delle scuole; di orientarli e formarli per un uso consapevole di tali strumenti.

\footnotetext{
2 I partecipanti erano studenti dei seguenti istituti: Istituto Professionale "Giovanni Falcone"; Istituto Tecnico "Lorenzo Lotto"; Istituto Professionale "Marigrazia Mamoli" e Liceo delle Scienze Umane "Secco Suardo". La scelta di creare gruppi di discussione non omogenei è stata fatta per favorire la polarizzazione delle diverse narrazioni emergenti durante la conversazione.
} 
Sono stati poi prodotti dei digital storytelling dagli studenti delle scuole superiori, con la supervisione dei tutor studenti universitari. Questi digital storytelling, sono stati quindi utilizzati dai docenti delle scuole superiori per orientare gli studenti.

3) Attività di autovalutazione della capacità degli studenti di svolgere le professioni in uscita dai Corsi di Scienze dell'Educazione. In questa fase hanno avuto un ruolo centrale i percorsi di orientamento svolti tramite le tecnologie, in particolar modo la riflessione conseguente alla visione dei Digital storytelling. Questi sono stati poi utilizzati per l'autovalutazione dagli insegnanti delle scuole secondarie di secondo grado per orientare $\mathrm{i}$ loro studenti e dai docenti universitari, per l'autovalutazione degli studenti iscritti al primo anno.

4) Percorsi di formazione per studenti tutor (didattici e di tirocinio) e l'attivazione di servizi di peer-tutoring. Gli studenti selezionati, dopo la formazione, hanno acquisito le competenze specifiche relative alla costruzione di prodotti digitali e alla gestione del tutoring nei confronti degli studenti provenienti dalle scuole.

5) Percorsi di formazione per tutor: nel corso di studio di Scienze dell'educazione esistono già diverse collaborazioni con i tutor in relazione al supporto per il percorso di tirocinio. Dato che i tutor sono in rapporto con i docenti universitari e le organizzazioni territoriali, è stato realizzato un momento di autoformazione tra i soggetti coinvolti. Questa autoformazione è andata poi a definire i prodotti attesi; in particolare il portfolio delle competenze che possono costituire un ponte per il passaggio degli studenti dagli Istituti Professionale al corso di Scienze dell'Educazione. Il criterio metodologico che si è seguito nei nostri interventi, è stato l'analisi dei repertori culturali delle studentesse e più precisamente delle loro narrazioni. Il percorso non ha inteso declinare la rilevazione delle narrazioni su un registro valutativo, bensì essere chiarificatore di alcuni possibili processi di costruzione sociale impliciti nelle narrazioni delle studentesse che si iscrivono a Scienze dell'educazione (Berger \& Lukmann, 1969). Si è teoricamente assunto il principio che le narrazioni siano la catena dei significanti che compongono le possibilità sintattiche-espressive delle studentesse, dei docenti di scuola superiore e dei docenti universitari, nonché della rete degli enti territoriali. È, se si vuole, l'orizzonte delle capacità espressive di chi ha partecipato al progetto, perciò si è scelto il digital storytelling al fine di mettere in scena le narrazioni che compongono la scelta universitaria e professionale delle studentesse.

La trama narrativa si costruisce attivamente, e in modo largamente inconsapevole all'interno di relazioni sociali e processi di socializzazione. Non la si può ridurre in formule di principio o di valore («Nella nostra scuola si 
lavora pensando innanzitutto allo studente» $\mathrm{o}$ «Nella nostra Università si persegue l'eccellenza»). Non che tali formule siano fasulle, tuttavia esse non bastano a render conto della trama discorsiva, anzi, finiscono con il delegarla a una funzione rappresentativa di cornice. E invece la narrazione è in atto, e il suo agire è alla base dei modi di immaginare il legame sociale e la relazione tra studenti e tra studenti e docenti, nello specifico delle espressioni soggettive di chi studia e di chi insegna nei contesti scolastici presi in esame e nell'università (Foucault, 1971).

La potenza della narrazione sta nella sua irriducibilità semiotica: non lo si può mai dire del tutto, proprio perché ogni dire è già l'esito della sua azione. Per contro, una rilettura continua e condivisa tra i ricercatori ha reso possibile individuare alcune faglie, attraverso cui ricostruire la trama discorsiva e riprodurla attraverso strumenti audiovisivi; faglie che sono espressione delle incertezze, delle esitazioni, dei desideri, delle preoccupazioni delle studentesse e degli studenti che si iscrivono a Scienze dell'educazione. La presa di parola del soggetto è, infatti, sia un'assunzione di responsabilità (sono soggetto di...), sia un'assunzione di parzialità (sono soggetto a...). La presenza di una trama discorsiva specifica, del suo dominio semantico in espressioni solo apparentemente neutrali, ma raffigurano il profondo nesso tra la dimensione affettiva delle studentesse e lo specifico contesto socio-organizzativo dell'Università, come luogo di relazioni intersoggettive e di apprendimento (Lakoff \& Johnson, 1980).

\section{Descrizione del percorso con gli studenti tutor nell'ambito del pro- getto POT: l'uso clinico del digital storytelling}

Le attività relative ai percorsi di orientamento (punto 2), all'autovalutazione (punto 3), al peer-tutoring (punto 4), hanno avuto come protagonisti principali nove studenti del corso di laurea in Scienze dell'Educazione, provenienti da quattro Istituti Superiori di Bergamo e Provincia, due istituti professionali, un istituto tecnico e un liceo.

Gli studenti, accompagnati da un formatore-tutor dell'Università degli Studi di Bergamo, sono stati formati come facilitatori dell'orientamento per la scelta del percorso di studi universitario e per la prefigurazione professionale.

Il percorso si è caratterizzato per la centralità che è stata assegnata agli studenti, al processo come strumento di lavoro, in particolare per l'utilizzo della narrazione digitale, e per aver utilizzato il dialogo e lo scambio tra i soggetti per generare conoscenza e supporto reciproco.

Gli studenti non sono stati coinvolti come destinatari di un lavoro di 
ricerca, ma come soggetti in azione di ricerca. Il loro protagonismo, secondo il modello della peer education, si è concretizzato non solo nell'essere al centro della prima fase, quella della loro formazione come tutor, ma in tutte le fasi del processo, compresa quella di costruzione del percorso per gli studenti delle scuole superiori. Quest'ultimo non è stato progettato solo dal formatore, ma è stato il prodotto finale del lavoro di osservazione che gli studenti hanno fatto del proprio percorso, dei loro bisogni, delle condizioni di ciascuno, della condivisione in gruppo delle riflessioni e della traduzione di queste in strumenti. Il ruolo del formatore è stato quello di stimolare, moderare e orientare le riflessioni per la costruzione di un metodo.

Nella sperimentazione del percorso di orientamento, come accennato, più che ai contenuti, si è data importanza al processo costruito attorno agli interrogativi emersi sia dagli studenti delle scuole superiori sia da quelli universitari. L'osservazione e la condivisione dei percorsi di vita delle persone nella scelta del corso di studi hanno fatto emergere i dubbi e hanno evidenziato oggetti di lavoro su cui la Scuola e l'Università possono lavorare nella costruzione di percorsi e strumenti di orientamento che coinvolgano anche il mondo del lavoro.

Inoltre, il lavoro di continua rilettura di quanto veniva fatto e di analisi dei dati raccolti durante gli incontri con il formatore è servito per la definizione dei passi successivi nell'ottica della costruzione di un metodo organizzativo replicabile.

La formazione degli studenti universitari, così come gli incontri con gli studenti delle scuole superiori, ha visto la realizzazione di attività che prevedevano l'utilizzo dello strumento narrativo, in particolare con il supporto delle tecnologie digitali: la restituzione del racconto di sé, del proprio vissuto e delle proprie prospettive, è servita per la riflessione e per la condivisione. Quest'ultima ha determinato l'efficacia del percorso di orientamento che ha messo al centro l'incontro tra persone di età diverse, pur prossime, e portatrici di esperienze originali, nel contesto di un percorso di studi e professionale simile. La possibilità di un dialogo aperto tra pari ha generato uno scambio e mutuo aiuto grazie alla comprensione reciproca dei bisogni.

$\mathrm{Ne}$ è conseguito un ruolo per il digital storytelling diverso, e per certi versi più ricco, rispetto a quello che assume di norma in campo educativo.

Di solito, infatti, il digital storytelling viene usato innanzitutto per la potenzialità di stimolare pubblici in contesti educativi formali, non formali e informali, nei quali può attivare processi riflessivi tramite la narrazione digitale (Petrucco, 2015): la valenza educativa della narrazione per i suoi destinatari (Bettelheim, 1976; Bruner, 1986) viene enfatizzata dal ricorso allo strumento digitale. Al digital storytelling viene riconosciuta, in secondo luogo, la capacità di stimolare la creatività dei soggetti coinvolti in prima 
persona nel progetto e sviluppo di narrazioni digitali promuovendo in loro competenze di vario tipo, da quelle narrative (Cassell \& Ryokai, 2001) a quelle scientifiche (Albano, Dello Iacono \& Mariotti, 2016), da quelle relazionali e di cittadinanza (Baschiera, 2014) a quelle sociali attraverso il lavoro cooperativo (Di Blas, Paolini \& Sabiescu, 2012).

Nell'ambito del progetto POT, il digital storytelling è stato sicuramente usato per fini del primo tipo, dal momento che le narrazioni digitali sono state sfruttate come strumento da usare nelle azioni di orientamento; e certamente si sono avute ricadute del secondo tipo sugli studenti che hanno partecipato attivamente alla co-costruzione delle narrazioni digitali. Ma c'è anche stato un uso che potremmo definire clinico del digital storytelling, nel momento in cui le sedute di registrazione sono servite per elicitare conoscenza, registrare esperienze, mettere a fuoco desideri, speranze e paure dei ragazzi coinvolti nelle discussioni ${ }^{3}$.

Il percorso è stato documentato in un video a cui è stato dato il titolo "Ad occhi aperti verso il futuro - Riflettere e conoscere per saper immaginare e decidere la professione di domani" suddiviso in tre capitoli che corrispondono ai tre temi chiave sui quali gli studenti hanno lavorato. Lo scopo del documentario è quello innanzitutto di documentare il processo ma anche quello di veicolare la conoscenza che il percorso ha prodotto non tanto dal punto di vista dei contenuti, per i quali non è stata avanzata alcuna pretesa di esaustività, quanto dal punto di vista metodologico, in termini di potenzialità e come contributo alla riflessione sull'organizzazione dei percorsi di orientamento.

Il primo capitolo del documentario, richiamando l'attività proposta dal formatore allo scopo di rendere gli studenti consapevoli del percorso di crescita svolto, riporta il confronto che è nato tra loro. La prima fase del percorso è servita per far emergere tra gli studenti universitari i propri vissuti, ricordando le scelte compiute e le motivazioni ad esse sottese; mettere a fuoco il cambiamento e le condizioni che l'hanno favorito o impedito; condividere le esperienze nel contesto universitario, le prospettive future, $\mathrm{i}$ desideri e i timori.

Lo sforzo di memoria, riflessione e condivisione è stato utile anche per perfezionare e completare l'attività proposta dal formatore come strumento per l'incontro con gli studenti delle scuole superiori, rappresentato nel

\footnotetext{
${ }^{3}$ Per lo sviluppo dei prodotti digitali di storytelling sono state utilizzate le piattaforme Google Meet per le videoconferenze, Padlet e Google Drive per la collaborazione sincrona e asincrona; per la produzione di immagini video e fotografiche sono state utilizzate videocamere professionali e smartphone, rispettivamente per le riprese dal vivo e per la produzione di materiali da trasmettere telematicamente; per la postproduzione video è stata utilizzata la Suite di Adobe.
} 
secondo capitolo del documentario. "Io non so cosa farò da grande" sintetizza gli incontri avvenuti in via telematica tramite la piattaforma Meet in cui gli studenti tutor hanno aiutato gli studenti delle scuole superiori ad aumentare la consapevolezza di sé rispetto alla sfera dello studio e del lavoro, degli obiettivi e prospettive. Gli incontri avvenuti, a partire dalle testimonianze degli studenti universitari e le domande loro poste, sono stati utili per far emergere dubbi, timori e curiosità e orientare gli studenti nella scelta del percorso di studi universitario.

Il tema della prefigurazione professionale ha trovato spazio nell'incontro con i professionisti dell'educazione realizzato allo scopo di orientare gli studenti verso le professioni a cui si può accedere con la laurea in Scienze dell'educazione. In "A un passo dal domani”, terzo capitolo del documentario, gli studenti universitari, intervistando i professionisti, non soltanto fanno emergere informazioni e un quadro complessivo utile a loro e agli studenti delle scuole superiori presenti ma, con le loro domande, danno evidenza di ciò che non conoscono e desiderano conoscere, offrendo spunti importanti ai fini della loro autovalutazione.

Il percorso documentato, sorpreso nel suo sviluppo dalla pandemia di, ha dovuto adattarsi alle circostanze e in esse trovare slancio per sperimentare nuove forme di relazione, azione e narrazione. Gli incontri tra studenti universitari e studenti degli istituti superiori, inizialmente realizzati in presenza, si sono trasferiti online, con la conseguente rimodulazione degli strumenti di lavoro in modalità interamente digitale. Il percorso, che proprio nella tecnologia digitale ha individuato uno strumento privilegiato per riflettere, incontrare e immaginare, ha saputo, con naturalezza, mantenere vivo il ritmo partecipativo delle persone coinvolte.

\section{Conclusioni}

Il progetto "Ad occhi aperti verso il futuro" ha messo in luce alcuni aspetti qualificanti di un percorso di orientamento e tutoraggio: il valore delle narrazioni e dei più ampi repertori culturali come interfaccia tra il mondo interiore della persona e le possibilità $\mathrm{o} i$ vincoli dei contesti accademici e professionali; il contributo delle nuove tecnologie nella costruzione di un vocabolario condivisibile tra le generazioni e i differenti ruoli sociali in gioco nei percorsi accademici e professionali; l'efficacia del peer-tutoring nell'accompagnamento degli studenti e delle studentesse definibili come metafragili. Ulteriori approfondimenti potranno arricchire il quadro complessivo, ad esempio: studiando il rapporto tra capitale sociale, economico e culturale e scelte universitarie, abbandoni e difficoltà occupazionali; o ancora studiando 
gli effetti che l'attuale didattica a distanza avrà sui processi di socializzazione e di scelta delle nuove generazioni.

La complessità, paradigma abusato, o malamente usato, nel recente passato, ha fatto irruzione nelle nostre case, nei nostri corpi, nella percezione del futuro. Mai come oggi ci rendiamo conto dell'interconnessione tra eventi apparentemente minimi (il famoso, e spesso banalizzato "effetto farfalla" che si materializza in un "effetto pipistrello e pangolino") e le ricadute globali che, nell'attuale situazione pandemica, divengono glocali, processuali, corporee, intime. Un senso di violabilità che obbliga a scelte immediate, ad incerte prossimità, ad una riconfigurazione del futuro.

Proprio su questo aspetto prefigurativo in tempi così complessi si gioca l'efficacia nel tempo del metodo del progetto ma, soprattutto, delle trasformazioni che le organizzazioni, a partire da quella universitaria, vorranno attuare proprio a partire dallo storico punto di svolta rappresentato dalla complessità della pandemia. Saremo in grado, parafrasando Mezirow e le teorie della complessità, di offrire opportunità di ri- e pre-configurazione proprio mentre riconfiguriamo le nostre cornici di riferimento? Potremo costruire dialoghi interni alle organizzazioni finalizzati alla trasformazione organizzativa e alla definizione di nuove prospettive di significato? In fondo, il "dilemma disorientante" da cui far scaturire questi processi ci è stato ampiamente e tragicamente presentato.

\section{Bibliografia}

Albano, G., Dello Iacono, U., \& Mariotti, M. A. (2016). L'argomentazione in matematica: la mediazione attraverso il digital interactive storytelling.Form@re, 16(1), pp. 105-115.

Barbier, R. (1996). La recherche-action. Paris: Anthropos.

Barbier, R. (1983). La recherche-action existentielle. Pour, La recherche-action, 90, juin-juillet, pp. 27-31.

Baschiera, B. (2014). L'uso del Digital storytelling in contesti di apprendimento cooperativo per l'inclusive education e l'acquisizione delle competenze chiave di cittadinanza. Formazione \& Insegnamento, XII(3), pp. 181-188.

Berger, P., \& Luckmann, T. (1966). The Social Constrution of Reality: a Treatise in the Sociology of Knowledge. New York: Doubleday (trad. it.: La realtà come costruzione sociale. Bologna: il Mulino, 1969).

Bertin, G. M., \& Contini, M. G. (1983). Costruire l'esistenza. Roma: Armando.

Bettelheim, B. (1976). The uses of enchantment: the meaning and importance of fairy tales. New York, USA: Knopf.

Bocchi, G., \& Ceruti, M. (2004). Educazione e globalizzazione. Milano: Raffaello Cortina. 
Bruner, J. (1986). Actual minds, possible worlds. Cambridge, USA: Harvard University Press.

Canevaro, A. (1976). I bambini che si perdono nel bosco: identità e linguaggi nell'infanzia. Firenze, Italy: La nuova Italia.

Cassell, J., \& Ryokai, K. (2001). Making space for voice: technologies to support children's fantasy and storytelling. Personal and Ubiquitous Computing, 5(3), pp. 169-190.

Ceruti, M., \& Bellusci, F. (2020). Abitare la complessità. Milano-Udine: Mimesis.

Di Blas, N., Paolini, P., \& Sabiescu, A. G. (2012). Collective digital storytelling at school: a whole-class interaction. International Journal of Arts and Technology, 582(4), pp. 271-292.

Fabbri, L., \& Romano, A. (2017). Metodi per l'apprendimento trasformativo. Roma: Carocci.

Foucault, M. (1971). L'ordre du discours. Paris: Gallimard (trad. it.: L'ordine del discorso e altri interventi. Torino: Einaudi, 2004).

Lakoff, G., \& Johnson, M. (1980). Metaphors We Live By. Chicago: University of Chicago Press (trad. it.: Metafora e vita quotidiana. Milano: Bompiani, 1998)

Mezirow, J. (2016). La teoria dell'apprendimento trasformativo. Milano: Raffello Cortina.

Openpolis (2020). La mobilità sociale passa da un'istruzione di qualità per tutti -«testo disponibile al sito: https://www.openpolis.it/la-mobilita-sociale-passa-daunistruzione-di-qualita-per-tutti/», ultima consultazione 7 febbraio 2021.

Petrucco, C. (2015). Digital storytelling as a reflective practice tool in a community of professionals. European Journal of Open, Distance and E-learning, 18(2), pp. 112-128.

Rai Radio 3 (2021). Uomini e profeti. Trasmissione del 31 gennaio.

Save the children (2020). I giovani al tempo del coronavirus, -- «testo disponibile al sito: https://s3.savethechildren.it/public/files/uploads/pubblicazioni/i-giovaniai-tempi-del-coronavirus.pdf », ultima consultazione 7 febbraio 2021.

Taleb, N. N. (2013). Antifragile. Milano: Il Saggiatore. 